\title{
Efficacy of inferior turbinate coblation for treatment of nasal obstruction
}

\author{
S E J FARMER, S M QuinE*, R ECCLES
}

\begin{abstract}
Objective: To determine the efficacy of inferior turbinate coblation for the treatment of nasal obstruction. Methods: Twenty patients awaiting submucosal diathermy to the inferior turbinates were recruited into the study. All underwent inferior turbinate coblation. Pre-operative and post-operative nasal function was investigated using posterior rhinomanometry and subjective symptom scales.

Results: There was no significant increase in nasal conductance two weeks after inferior turbinate coblation $(p=0.159)$. However, three months after inferior turbinate coblation, median nasal conductance had increased significantly, from 203 to $324 \mathrm{~cm}^{3} / \mathrm{s}(p=0.004)$. The median increase in nasal conductance was $73 \mathrm{~cm}^{3} / \mathrm{s}$ or 43.5 per cent. Post-operative visual analogue patients' reported post-operative visual analogue scales scores for nasal obstruction decreased significantly, both two weeks $(p=0.006)$ and three months after inferior turbinate coblation $(p=0.001)$ when compared to Preoperative values. There was no change in the reported severity of rhinorrhoea, nasal itching or sneezing. There was a significant relationship $(\rho=-0.57, p=0.014)$ between pre-operative nasal conductance and change in nasal conductance after inferior turbinate coblation.

Conclusions: This study confirms the short-term efficacy of inferior turbinate coblation for the treatment of nasal obstruction. The benefit was greatest in patients with lower pre-operative nasal conductance. Objective measures of nasal obstruction may be important when selecting patients for inferior turbinate coblation.
\end{abstract}

Key words: Nasal Obstruction; Turbinates; Electrosurgery

\section{Introduction}

Nasal obstruction due to inferior turbinate enlargement is a common problem. There are many surgical treatments for turbinate enlargement, but little objective evidence to support their efficacy. Inferior turbinate coblation is a relatively new, submucosal technique that involves the use of a bipolar wand and a standard electrosurgical unit known as the Coblator ${ }^{\circledR}$ surgery system (ArthroCare, Harrogate, $\mathrm{UK}$ ). An electrically conductive fluid is employed in the gap between the electrode and the tissue. It is claimed that this results in the "non-thermal volumetric removal of tissue'. To date, few published studies have reported the use of inferior turbinate coblation for the treatment of nasal obstruction. ${ }^{2-4}$ Subjective improvement in the frequency and severity of nasal obstruction has been reported following inferior turbinate coblation. ${ }^{2,3}$ However, there is conflicting evidence concerning the objective improvement in nasal obstruction after inferior turbinate coblation. ${ }^{2}$

It is important that surgical efficacy is determined using an objective measure of nasal obstruction.
This is because the subjective sensation of nasal airflow is influenced by many factors, including mood, cold receptors and congestion of the ostia of the paranasal sinuses. ${ }^{5}$ Surgery aims simply to unblock the nose, so it seems entirely appropriate that an objective measurement of nasal airflow is used for evaluation. This study evaluated the efficacy of inferior turbinate coblation using posterior rhinomanometry to provide measurements of total nasal conductance of airflow. Rhinomanometry is generally accepted as the 'gold standard' technique for assessing nasal obstruction. ${ }^{6}$ Posterior rhinomanometry has the advantage of enabling direct measurement of total nasal conductance of airflow, and also permits measurement when there is total unilateral airway obstruction.

\section{Methods}

\section{Ethical considerations}

The study was approved by the south east Wales research ethics committee and the Cardiff and Vale

From the Common Cold Centre, Cardiff School of Biosciences, Cardiff University, and the *Department of Otolaryngology, Head and Neck Surgery, University Hospital of Wales, Cardiff, Wales, UK.

Presented as a poster at the British Rhinology Society 6th Annual Meeting, 25th May 2007, London, England, and in full at the Welsh Otorhinolaryngological Association Meeting, 26th October 2007, Swansea, Wales, UK.

Accepted for publication: 10 April 2008. First published online 9 June 2008. 
National Health Service trust research and development office. All patients provided informed consent in order to participate.

\section{Participants}

All patients on the University Hospital of Wales waiting list for submucosal diathermy to the inferior turbinates were invited to take part in the study. Patients aged 18 years or more with nasal obstruction as their main nasal symptom and with no previous history of nasal surgery were eligible for inclusion. Patients were excluded if they had a severe septal deviation or other condition that could cause nasal obstruction, such as nasal polyps, or if they were drug or alcohol abusers.

Patients who fulfilled the entry criteria for the study were assessed using subjective symptom severity scores and posterior rhinomanometry. Patients were scheduled for inferior turbinate coblation and were reviewed two weeks and three months postoperatively. Participants provided symptom severity scores and had their nasal conductance measured at each post-operative visit.

\section{Objective and subjective measurements of nasal obstruction}

Nasal obstruction was measured using posterior rhinomanometry and symptom severity scale scores. Patients completed their symptom severity scores before having their nasal conductance measured in order to avoid bias. Patients were asked to refrain from consuming menthol-containing medications and more than four units of alcohol for 24 hours prior to each assessment. They were also requested to avoid oral and nasal decongestants for 48 hours prior to each assessment, and nasal steroids and antihistamines for two weeks before each assessment. To avoid other confounding factors in the measurement of nasal obstruction, patients who had had an upper respiratory tract infection within two weeks of the assessment had their appointment rescheduled.

\section{Posterior rhinomanometry}

Total nasal conductance of airflow was measured using posterior rhinomanometry using the NR6-2 rhinomanometer (GM Instruments, Glasgow, Scotland, UK) at a sample pressure of $75 \mathrm{~Pa}$. The rhinomanometer was calibrated daily. Participants were asked to gently blow their nose prior to each measurement to clear any secretions. Participants were trained to breathe into the facemask whilst sealing their lips around the pressure sensing tube in the oral cavity. Participants were asked to breathe at a normal rate and depth, and two consecutive sets of four respiratory cycles were obtained. After the first set of four breaths was completed, the participant was requested to rest, remove the facemask and replace it again before delivering the second set of breaths. The coefficient of variation $(\mathrm{CV})$ was calculated after the second set of breaths, and patients were required to achieve a $\mathrm{CV}$ of 10 per cent or less. The mean nasal resistance of the eight respiratory cycles was than calculated.
Participants were given three attempts to achieve a $\mathrm{CV}$ of 10 per cent or less. Nasal conductance of airflow was calculated by dividing the sample pressure by the mean total nasal resistance. Patients who presented with complete nasal obstruction were deemed to have a nasal conductance of $0 \mathrm{~cm}^{3} / \mathrm{s}$.

\section{Symptom severity scales}

A $100 \mathrm{~mm}$ visual analogue scale (VAS) anchored by the descriptors 'nose completely clear' $(0 \mathrm{~mm})$ and 'nose completely blocked' $(100 \mathrm{~mm})$ was used to assess present subjective severity of nasal obstruction. The severity of nasal obstruction over the past week was scored on a four-point ordinal scale (symptom not present $=$ zero, mild symptoms $=$ one, moderate symptoms $=$ two and severe symptoms $=$ three). The subjective severities of rhinorrhoea, nasal itching and sneezing over the previous week were also scored on a four-point ordinal scale.

\section{Operative technique}

The surgery was performed in accordance with a standard operating procedure, by two surgeons (SEJF and SMQ). Guided by the advice of the attending doctor, participants were given the option to have the surgery performed under local or general anaesthesia in the operating theatres at the University Hospital of Wales, Cardiff.

The patient was positioned supine with $30^{\circ}$ of head elevation. The nose was prepared using a standard technique with the application of cocaine paste, and each inferior turbinate was infiltrated with 2 per cent lidocaine with 1:80 000 adrenaline.

The surgery was performed using a Coblator II surgery system and a ReFlex Ultra ${ }^{\mathrm{TM}} 45$ wand (ArthroCare) set at power level four. The wand was activated and the anterior end of the inferior turbinate was pierced with the wand tip. The wand was then advanced submucosally, whilst still activated, to the second or third marker, depending on the size of the turbinate, to create a tissue channel. Tensecond periods of activation were performed at each marker depth to create a series of two or three lesions, depending on the depth of insertion. A further one or two channels were created, depending on the size of the inferior turbinate.

Post-operatively, patients were recommended to use analgesia as necessary and were provided with a routine prescription. They were advised to use regular saline douches and to discontinue all other topical nasal medication.

\section{Statistical analysis}

Statistical analysis of results was performed using Wilcoxon's test. Spearman's rank correlation coefficient was used to determine the relationship between variables. A $p$ value of $\leq 0.05$ was deemed to be statistically significant.

Where results are presented as box plots, the median value is indicated by a thick black line. The interquartile range is demonstrated by a shaded box ranging from the 25 th to the 75 th percentile. Whiskers 
at the end of the box are constructed according to Tukey's method; they show the largest and smallest observed values that are less than 1.5 box lengths from either end of the box.

\section{Results}

Twenty patients were recruited into the study. One patient was excluded during the follow-up period because he developed nasal polyps. Of the 19 patients included in the study, 18 had inferior turbinate coblation performed under general anaesthesia. One patient had coblation performed under local anaesthesia due to suspicion of malignant hyperthermia. One patient failed to attend for his three-month follow-up appointment, but his two-week follow-up results were included in the study.

The patients comprised 14 men ( 74 per cent) and five women (26 per cent). The mean age of the study population was 32 years (range 19 to 59 years). Five patients ( 26 per cent) had documented evidence of allergy in the form of positive skin prick tests to commonly inhaled allergens.

Two weeks after inferior turbinate coblation, 13 patients ( 68 per cent) had an increased nasal conductance of airflow. By three months post-operatively, 15 patients (83 per cent) had an increased nasal conductance of airflow. The median pre-operative nasal conductance was $203 \mathrm{~cm}^{3} / \mathrm{s}$ (range $0-615 \mathrm{~cm}^{3} / \mathrm{s}$, Figure 1). The median nasal conductance two weeks after inferior turbinate coblation was $250 \mathrm{~cm}^{3} / \mathrm{s}$ (range $175-475 \mathrm{~cm}^{3} / \mathrm{s}$ ); three months after surgery, it was $324 \mathrm{~cm}^{3} / \mathrm{s}\left(90-560 \mathrm{~cm}^{3} / \mathrm{s}\right)$. There was no significant difference in nasal conductance two weeks after surgery compared with pre-operative values $(p=0.159)$. Three months after surgery, nasal conductance was significantly greater than pre-operative values $(p=0.004)$. The

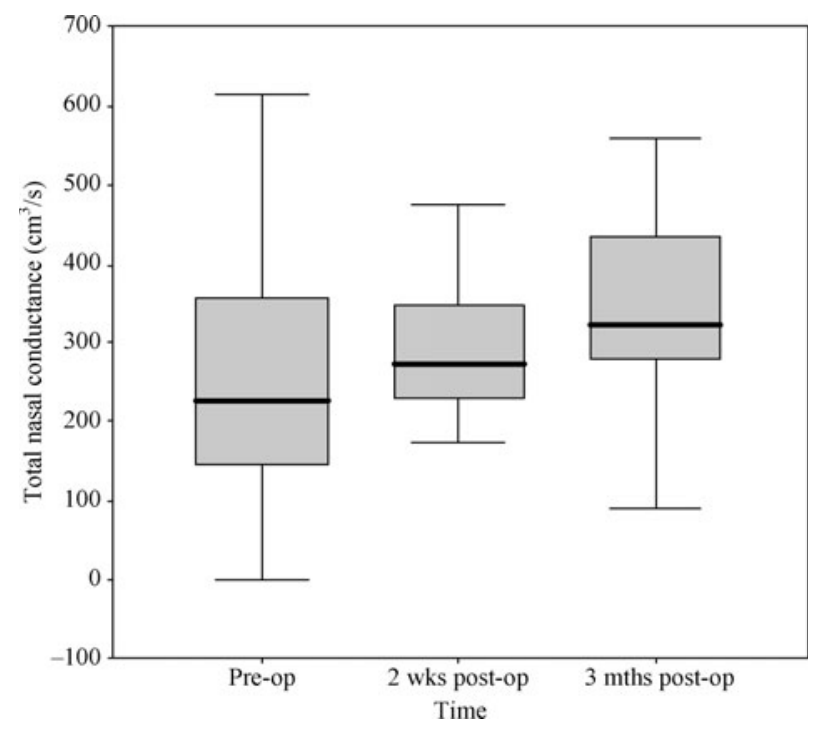

FIG. 1

Effect of inferior turbinate coblation on total nasal conductance of airflow. See text for explanation of boxes and whiskers. Pre-op $=$ pre-operative; post-op $=$ post-operative; wks $=$ weeks; mths $=$ months median change in nasal conductance three months after coblation was an increase of $73 \mathrm{~cm}^{3} / \mathrm{s}$ (range -108 to $279 \mathrm{~cm}^{3} / \mathrm{s}$ ) or 43.5 per cent (range -18 to 279 per cent).

Fifteen patients (79 per cent) reported lower VAS scores two weeks after surgery, compared with preoperative scores. Three months after coblation, 14 patients (78 per cent) reported lower VAS scores for nasal obstruction, compared with pre-operative scores. The median VAS score for nasal obstruction before inferior turbinate coblation was $78 \mathrm{~mm}$ (range 29-100 mm, Figure 2). Two weeks after surgery, the median VAS score was $50 \mathrm{~mm}$ (range $0-81 \mathrm{~mm}$ ); three months after surgery, it was $31.5 \mathrm{~mm}$ (range $5-81 \mathrm{~mm}$ ). The VAS score for nasal obstruction reported two weeks after coblation was significantly lower than the pre-operative score $(p=0.006)$, as was the VAS score for nasal obstruction reported three months after surgery $(p=0.001)$.

The VAS scores for nasal obstruction reported two weeks and three months after coblation were significantly lower than the pre-operative scores $(p=$ 0.046 and $p=0.001$, respectively). There was no significant change in the reported severity of rhinorrhoea, nasal itching or sneezing following inferior turbinate coblation.

There was a significant relationship between preoperative nasal conductance of airflow and change in nasal conductance of airflow three months after inferior turbinate coblation (Figure 3). Spearman's correlation coefficient was $-0.57(p=0.014)$.

\section{Discussion}

Cottle $^{7}$ recognised the importance of obtaining objective measurements of nasal obstruction back in the 1960s, and concluded that '... just as clearly

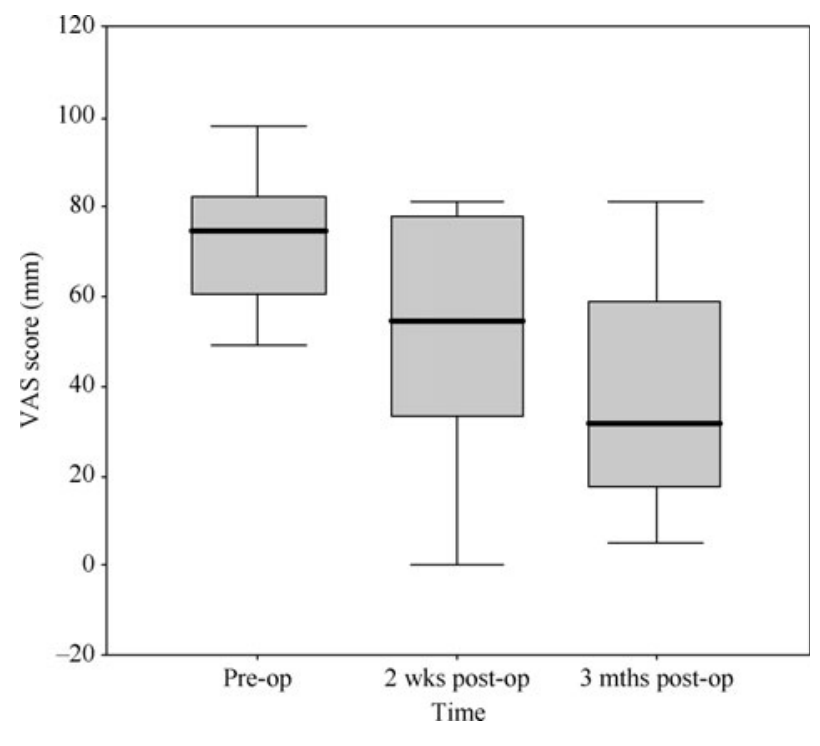

FIG. 2

Effect of inferior turbinate coblation on VAS score for nasal obstruction. See text for explanation of boxes and whiskers. Pre-op $=$ pre-operative; $\quad$ post-op $=$ post-operative; $\quad$ wks $=$ weeks; $\mathrm{mths}=$ months 


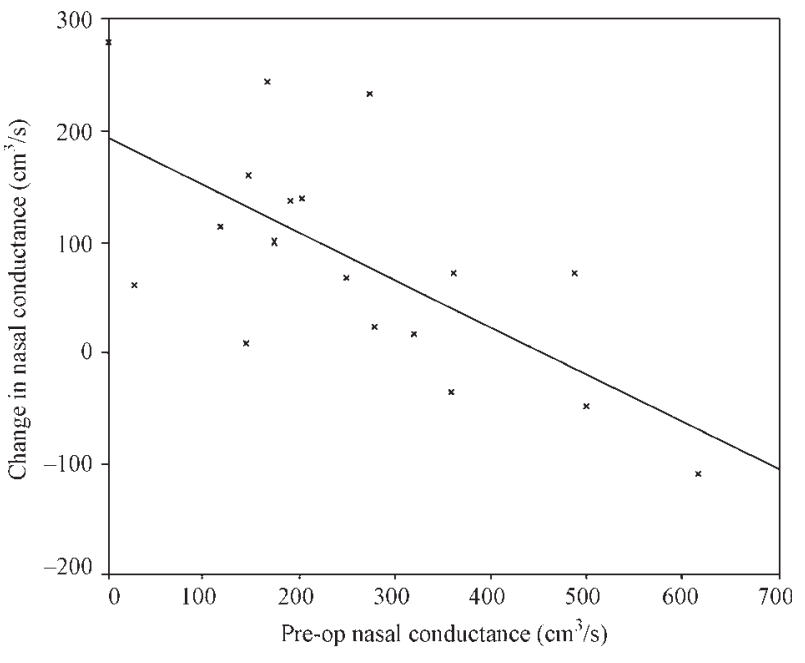

FIG. 3

Relationship between pre-operative (pre-op) nasal conductance of airflow and change in nasal conductance of airflow three months after inferior turbinate coblation.

as the ear surgeon needs audiometry pre- and post-operatively, the nose surgeon needs rhinomanometry'. Unfortunately, there continues to be little objective evidence for the efficacy of surgical treatments for nasal obstruction. Back et al. ${ }^{2}$ attempted to objectively assess the outcome of inferior turbinate coblation in a group of 20 patients with nasal obstruction, using anterior rhinomanometry and acoustic rhinometry. However, although there was an increase in nasal cavity volumes six months and one year after inferior turbinate coblation, there was no difference three months after the surgery. There was no significant difference in nasal conductance after inferior turbinate coblation. Given these findings, the objective evidence concerning the efficacy of inferior turbinate coblation is unclear.

Total nasal conductance of airflow is the most relevant functional measurement of the airway ${ }^{8}$ and is best measured with posterior rhinomanometry. Anterior rhinomanometry, calculated from unilateral values using Ohm's law of parallel resistors, cannot measure total nasal conductance of airflow. The validity of this technique has been questioned due to a lack of similarity between calculated and measured values. ${ }^{9}$

The present study showed a significant improvement in median total nasal conductance three months after inferior turbinate coblation, equivalent to a median increase in nasal conductance of airflow of 43.5 per cent. This conflicts with the findings of the only other published study ${ }^{2}$ addressing the objective results of turbinate coblation surgery. This may be explained by differences in the techniques used to obtain the objective measurements. Back et al. ${ }^{2}$ not only used anterior rhinomanometry, as opposed to the posterior rhinomanometry used in our study, but also failed to clearly describe their technique for obtaining measurements. The median nasal resistance of patients in Back and colleagues' study was less than $0.20 \mathrm{~Pa} / \mathrm{cm} /$ second. $^{2}$ This is within the normal range, which is typically reported to be less than $0.23 \mathrm{~Pa} / \mathrm{cm} /$ second. $^{10}$ This raises issues about patient selection for this study. In comparison, in our study the median nasal conductance was $203 \mathrm{~cm}^{3} / \mathrm{s}$, which is equivalent to a nasal resistance of $0.37 \mathrm{~Pa} / \mathrm{cm} /$ second.

There was no significant difference in nasal conductance of airflow, comparing pre-operative and early post-operative (two-week) assessments. This may be due to the small number of patients. Few other studies have examined the objective benefit of turbinate electrosurgery in the early postoperative period. Rhee et al. ${ }^{11}$ operated upon 16 patients using radiofrequency tissue reduction, and used rhinomanometry to measure nasal resistance at days one, two and three and weeks one, four and eight post-operatively. They found no significant improvement, compared with pre-operative nasal resistance values, until eight weeks after surgery. This may be explained by the early tissue response to the trauma of surgery, which comprises an inflammatory phase, a lag phase and a proliferative phase. ${ }^{12}$ In the nasal mucosa, this characteristically results in oedema, rhinorrhoea and crusting during healing. Another potential explanation for the lack of objective improvement two weeks after coblation is that the surgery results in a progressive reduction in the volume of turbinate tissue post-operatively because fibrosis develops slowly.

This study demonstrated a significant improvement in patients' subjective scores for nasal obstruction two weeks after surgery, compared with pre-operative scores. This improvement was maintained three months post-operatively. Other studies have also shown subjective improvement in the severity of nasal obstruction after inferior turbinate coblation. ${ }^{2,3}$ Back et al. $^{2}$ used a similar $100 \mathrm{~mm}$ VAS but unfortunately did not report any values to enable a direct comparison with the present study.

There are a number of potential explanations as to why there was subjective improvement in nasal obstruction two weeks after inferior turbinate coblation but no improvement in nasal conductance of airflow. The small sample size may be responsible, or the multifactorial nature of the sensation of nasal airflow. Alternatively, the early subjective improvement in nasal blockage may be explained by the placebo effect of surgery. This explanation is supported by the fact that 68 per cent of patients experienced an improvement in nasal conductance two weeks after surgery, but that 79 per cent of patients experienced a subjective improvement in nasal obstruction. Patients seemed to over-rate the efficacy of the surgery two weeks post-operatively. Inclusion in this study might also bias subjective scores, if patients felt obliged to score in favour of inferior turbinate coblation. Alternatively, patients may perceive an improvement in nasal airflow due to a reduction in post-operative oedema as overall improvement in nasal obstruction when this was not the case. Underlying differences in objective and subjective scoring may also be relevant. Rhinomanometry provides a calibrated measure of nasal 
airflow. In contrast, the VAS is not standardised, although it references extreme values. Measurement is entirely based on patient opinion; thus, patients may have the same nasal conductance but their opinion on the severity of their nasal obstruction may differ, so that they record different VAS scores.

- There is little objective evidence to support the efficacy of surgical treatments for inferior turbinate enlargement

- This study aimed to evaluate the short-term efficacy of inferior turbinate coblation for the treatment of nasal obstruction

- There was a significant improvement in nasal conductance of airflow three months after inferior turbinate coblation

- Subjective improvements in nasal obstruction were also recorded after inferior turbinate coblation

- Patients with the lowest pre-operative nasal conductance gained most objective benefit from inferior turbinate coblation

Inferior turbinate coblation did not reduce the severity of sneezing, nasal itching or rhinorrhoea. This conflicts with the findings of Back et al., who showed a significant reduction in VAS scores for nasal discharge, itching, sneezing and crusting 12 months after coblation. ${ }^{2}$ The study sample size was similar to that in our study; the different result may be explained by chance, by the different VAS used, or by the different numbers of patients with allergic rhinitis included in each study. It seems unlikely that coblation should have an effect on the severity of nasal symptoms other than nasal obstruction, as it aims to reduce the size of the inferior turbinate. (However, it has been reported that electrosurgery may damage cholinergic nerves and potentially reduce glandular activity.) ${ }^{13}$ Bhattacharyya et l. $^{3}$ concur with the findings of the present study, reporting no significant improvement in the severity of postnasal discharge or mucus production after coblation surgery.

We found a statistically significant correlation between pre-operative nasal conductance and change in nasal conductance after inferior turbinate coblation. Put simply, patients with the most blocked noses experienced the greatest improvement in nasal conductance following inferior turbinate coblation. The small number of patients included in this study limits our ability to draw firm conclusions from the data. However, it appears that pre-operative nasal conductance could potentially be used to determine which patients are offered inferior turbinate coblation. It would be possible to determine a cut-off value for pre-operative nasal conductance with maximal sensitivity and specificity in predicting objective benefit from surgery. This study suggests that this cut-off is probably well above the lower limit for normal nasal conductance. The current method of selection for turbinate surgery is based on patient history and subjective assessment of the patient. This may explain why reports on the efficacy of turbinate surgery have been so variable. This study has shown that if patients were selected for surgery according to objective pre-operative criteria, then the potential for improvement in nasal conductance would be greater. The significant correlation between pre-operative nasal conductance and change in nasal conductance after inferior coblation surgery only serves to reinforce the importance of obtaining objective measurements of nasal obstruction when assessing patients with this complaint.

\section{Conclusion}

This study provides the first convincing evidence to support the short-term efficacy of inferior turbinate coblation. Patients with the lowest pre-operative nasal conductance of airflow gain greatest objective benefit from turbinate coblation, and this has potential implications for the selection of patients for surgery. This finding also highlights the importance of obtaining objective measurements of nasal obstruction when assessing this symptom.

\section{References}

1 ArthroCare Corporation. http://www.arthrocareent.com/ wt/page/coblation_explained 2005 [1 October 2007]

2 Back LJ, Hytonen ML, Malmberg HO, Ylikoski JS. Submucosal bipolar radiofrequency thermal ablation of inferior turbinates: a long-term follow-up with subjective and objective assessment. Laryngoscope 2002;112:1806-12

3 Bhattacharyya N, Kepnes LJ. Clinical effectiveness of coblation inferior turbinate reduction. Otolaryngol Head Neck Surg 2003;129:365-71

4 Atef A, Mosleh M, El Bosraty H, Abd El Fatah G, Fathi A. Bipolar radiofrequency volumetric tissue reduction of inferior turbinate: does the number of treatment sessions influence the final outcome? Am J Rhinol 2006; 20: $25-31$

5 Quine SM, Eccles R. Nasal resistance from the laboratory to the clinic. Curr Opin Otolaryngol Head Neck Surg 1999 7:20-5

6 Eccles R. Evaluation of the nasal airway and nasal challenge. In: Kerr AG, ed. Scott-Brown's Otolaryngology, 6th edn. Oxford: Butterworth-Heinemann, 1997; 4/4/1-15

7 Cottle M. Concepts of nasal physiology as related to corrective nasal surgery. Arch Otolaryngol Head Neck Surg 1960; 72:11-20

8 Quine SM, Aitken PA, Eccles R. Effect of submucosal diathermy to the inferior turbinates on unilateral and total nasal airflow in patients with rhinitis. Acta Otolaryngol 1999;119:911-15

9 Panagou PS, Loukides S, Tsipra S, Syrigou K, Anastasakis C, Kalogeropoulos N. Evaluation of nasal patency: comparison of patient and clinician assessments with rhinomanometry. Acta Otolaryngol 1999;118:847-51

10 Eccles R. Nasal airflow in health and disease. Acta Otolaryngol 2000;120:580-95

11 Rhee CS, Kim DY, Won TB, Lee HJ, Park SW, Kwon TY et al. Changes of nasal function after temperaturecontrolled radiofrequency tissue volume reduction for the turbinate. Laryngoscope 2001;111:153-8

12 Aljafri AM, Kingsnorth A, eds. Fundamentals of Surgical Practice. London: Greenwich Medical Media, 1998

13 Talaat M, El-Sabawy E, Baky FA, Raheem AA. Submucous diathermy of the inferior turbinates in chronic hypertrophic rhinitis. J Laryngol Otol 1987;101: 452-60 
Address for correspondence:

Miss Sarah Farmer,

ENT Specialist Registrar

Common Cold Centre,

Cardiff School of Biosciences,

Cardiff University, Museum Avenue,

Cardiff CF10 3US, Wales, UK.
Fax: 02920874093

E-mail: farmers@cardiff.ac.uk

Miss S E J Farmer takes responsibility for the integrity of the content of the paper.

Competing interests: None declared 